\title{
The diversification benefits of hedge funds and funds of hedge funds
}

\author{
Maher Kooli \\ School of Business and Management, University of Quebec in Montreal (UQAM), CP 6192, \\ Succursale Centre-Ville, Montreal Quebec, Canada, H3C 4R2. \\ Tel: + 1514987 3000, ext. 2082; Fax: + 1514987 0422; E-mail: kooli.maher@uqam.ca \\ Received (in revised form): 7th September, 2006
}

Maher Kooli is a professor of finance at the School of Business and Management, University of Quebec in Montreal (UQAM). He holds a PhD in finance from Laval University (Quebec) and was a postdoctoral researcher in finance at the Center of Interuniversity Research and Analysis on Organisations. He also worked as a research advisor for la Caisse de Depot et Placement de Quebec (CDP Capital). His current research interests include alternative investments, initial public offerings and mergers and acquisitions.

\section{Practical applications}

The hedge funds (HF) and the funds of HF universe is evolving rapidly. To explain the growing attraction of these alternative investments, investors often rely on the existence of free lunch. The paper examines the diversification benefits of HF and funds of HF. We rely on mean-variance spanning tests to analyse this issue. It is found that including HF or fund of HF portfolios to a set of benchmark portfolios (US stocks only) provides an extra return for a unit increase in standard deviation. However, this conclusion is less evident when we consider an internationally diversified portfolio as a benchmark. Our message to portfolio managers is that the value added by HF is uncertain and depends upon the opportunity set that is considered.

\section{Abstract}

We examine whether investors can improve their investment opportunity set through the addition of a hedge fund (HF) or fund of HF portfolio to different sets of benchmark portfolios. Using data from 1994 to 2004, we find that HF, as an asset class, improve the mean-variance frontier of sets of benchmark portfolios sorted by firm size and book-to-market ratio. However, we find that the improvement comes mainly from a leftward shift of the global minimum-variance portfolio rather than the tangency portfolio. Furthermore, investors who already hold a diversified portfolio do not improve their investment opportunity set by adding HF portfolios. On the other hand, we find that investing in funds of HF, as an asset class, does bring diversification benefits for mean-variance investors, even when we enlarge our investment opportunity set by including fixed income, international assets and commodities. Derivatives Use, Trading \& Regulation (2007) 12, 290-300. doi:10.1057/palgrave.dutr.1850053

Keywords: hedge funds; funds of funds; meanvariance spanning test

\section{INTRODUCTION}

Hedge fund (HF) investment has increased dramatically in the last decade. For instance, Van
Derivatives Use, Trading \& Regulation Vol. 12 No. 4, 2007 pp. 290-300 (C) 2007 Palgrave Macmillan Ltd 1357-0927 \$30.00 
Hedge Fund Advisors International, Inc. reports a total of 8,100 global HF managing close $\$ 1.2$ trillion in capital at the end of year 2005. To explain the growing attraction of these funds, investors often rely on the existence of free lunch. Indeed, HF table higher average returns than standard market indexes and higher Sharpe ratio. More important, they tend to have lower correlations with most passive investments. Low correlation means that adding HF to a portfolio can actually reduce risk and still provide the benefits of higher average returns. However, these conclusions depend on the investor's ability to achieve the performance of passive investments. For those who do not meet the definition of accredited investor, investing in Funds of funds (FOF) is presently the only way to gain access to absolute return strategies. Indeed, FOF let investors with as little as $\$ 10,000$ to participate in a diversified mix of HF, while traditional $\mathrm{HF}$ require a minimum investment of $\$ 250,000$. FOF can offer the most attractive risk-adjusted rates of return with low to zero correlation to most traditional portfolios. However, the diversification across manager styles comes at the cost of a multiplication of the fees paid by the investor (Brown et al. ${ }^{1}$ ).

The purpose of this article is a better understanding of the diversification benefits of HF and funds of HF. We rely on mean-variance spanning tests to analyse this issue. Throughout the paper, we consider that a set of asset returns provides diversification benefits relative to a set of benchmark returns if adding these returns to the benchmark leads to a significant leftward shift in the mean-standard deviation frontier. We should, however, note that even if the analysis is based on historical returns, the benefits we find could have little bearing on future performance.
The rest of the paper is organised as follows: The next section sets out the methods used to evaluate mean-variance spanning; The subsequent section describes the data for HF, FOF and benchmark portfolios; The penultimate section discusses the empirical results and the final section concludes the paper.

\section{TESTING FOR DIVERSIFICATION BENEFITS}

HF (or FOF) can play an important role as a diversifier due to its low or negative correlation to other asset classes. This highlights the important role HF (or FOF) may play in the asset allocation mix as it protects the portfolio from undesirable moves in the equity market.

We rely on mean-variance spanning tests to evaluate the economic diversification gains of HF or FOF portfolios for investors (DeRoon and Nijman; ${ }^{2}$ Kan and Zhou ${ }^{3}$ ). These tests enable us to analyse the effect on the meanvariance frontier of adding new assets to a set of benchmark assets. For example, we can say that spanning occurs when the mean-variance frontier of the set of benchmark portfolios and that of the benchmark portfolios plus a HF portfolio (or a FOF portfolio) coincide. In this case, investors do not benefit from adding a HF (or a FOF) to their current portfolio.

In finite samples, the question of whether an observed shift is statistically significant can be tested using regression-based tests of meanvariance spanning.

\section{Asymptotic and finite sample test statistics}

In this section, we briefly describe the statistical tests used to examine whether adding a HF portfolio can significantly improve the investment 
opportunity set relative to a set of benchmark assets. For purposes of convenience, we follow the notation and treatment in Kan and Zhou.

We denote by $K$ the set of benchmark portfolios (ie, without HF or FOF portfolios) with return $R_{1 t}$ and by $N$ the set of test assets (the HF or FOF portfolio) with return $R_{2 t}$. We estimate the following model using ordinary least squares:

$$
\begin{gathered}
R_{2 t}=\alpha+\beta R_{1 t}+\xi_{t}, \quad t=1,2, \ldots T, \\
(R=X B+E \text { in matrix notation })
\end{gathered}
$$

Following Huberman and Kandel, ${ }^{4}$ the null hypothesis of 'spanning' is:

$$
H_{0}: \alpha=0_{N}, \delta=1_{N}-\beta 1_{K}=0_{N},
$$

where $0_{N}$ is defined as the zero vector of $N$ elements. We then denote by $\lambda_{1}$ and by $\lambda_{2}$ the two eigenvalues of the matrix $\hat{H} \hat{G}^{-1}$ (see Appendix A for the definition of $\hat{H}$ and $\hat{G}$ ). The distribution of the asymptotic Wald test statistic of the null hypothesis is:

$$
W=T\left(\lambda_{1}+\lambda_{2}\right) \sim \chi_{2 N}^{2}
$$

In this case, if we fail to reject the null hypothesis, then the benchmark assets span the mean-variance frontier of the benchmark plus a HF or FOF portfolio, that is investors are not able to enlarge their investment opportunity set by adding a HF or FOF portfolio. On the other hand, if the null hypothesis is rejected, adding a HF or FOF portfolio does improve the investment opportunity set.

The corresponding asymptotic likelihood ratio and Lagrange multiplier tests are:

$$
\begin{gathered}
L R=T \sum_{i=1}^{2} \ln (1+\lambda i) \sim \chi_{2 N}^{2} \\
L M=T \sum_{i=1}^{2} \frac{\lambda_{i}}{1+\lambda_{i}} \sim \chi_{2 N}^{2}
\end{gathered}
$$

As the Wald test is not the uniformly most powerful test, we also use the likelihood ratio and Lagrange multiplier tests for mean-variance spanning. The exact finite sample distribution of the likelihood ratio test under the null, as in Huberman and Kandel, is:

$$
\begin{aligned}
& \left(\frac{1}{U^{1 / 2}}-1\right)\left(\frac{T-K-N}{N}\right) \sim \\
& F_{2 N, 2(T-K-N)} \text { for } N=2 \\
& \left(\frac{1}{U^{1 / 2}}-1\right)\left(\frac{T-K-1}{2}\right) \sim \\
& F_{2,2(T-K-1)} \text { for } N=1
\end{aligned}
$$

where $U=|\hat{G}| /|\hat{H}+\hat{G}|$

In general, the test for mean-variance spanning can be divided into two parts: (1) the spanning of the global minimum-variance portfolio and (2) the spanning of the tangency portfolio. Therefore, we can re-write the Wald test as

$$
\begin{aligned}
W= & T\left(\frac{\left(\hat{\sigma}_{R_{1}}\right)^{2}}{\left(\hat{\sigma}_{R}\right)^{2}}-1\right) \\
& +T\left(\frac{1+\hat{\theta}_{R}\left(R_{1}^{G M V}\right)^{2}}{1+\hat{\theta}_{R} 1\left(R_{1}^{G M V}\right)^{2}}-1\right)
\end{aligned}
$$

where $R$ is the return of the benchmark assets plus the HF portfolio; $\hat{\sigma}_{R_{1}}^{2}$ and $\hat{\sigma}_{R}^{2}$ are the global minimum variance of the benchmark assets and that of the benchmark assets plus a HF or FOF portfolio, respectively; $\hat{\theta}_{R_{1}}\left(R_{1}^{G M V}\right)$ is the slope of the asymptote of the mean-variance frontier for the benchmark assets; and $\hat{\theta}_{R}\left(R_{1}^{G M V}\right)$ is the slope of the tangency line of the mean-variance frontier for the benchmark portfolios plus a HF or FOF portfolio.

The first term measures the change of the global minimum-variance portfolios due to the addition of a HF or FOF portfolio. The second term measures whether there is an improvement 
of the squared tangency slope after adding a HF or FOF portfolio to the set of benchmark portfolios.

Kan and Zhou suggest a step-down procedure, which requires us to first test $\alpha=0_{N}$, and then test $\delta=1_{N}-\beta 1 K=0_{N}$, conditional on $\alpha=0_{N}$.

The step-down asymptotic Wald tests can be written as:

$$
\begin{aligned}
& W_{1}=T\left(\lambda_{i}\right) \sim \chi_{N}^{2} \\
& W_{2}=T\left(\lambda_{i}\right) \sim \chi_{N}^{2}
\end{aligned}
$$

If the rejection is due to the first test, we conclude that it is because the two tangency portfolios are very different. If the rejection is due to the second test, we conclude that it is because the two global minimum-variance portfolios are very different.

\section{Spanning tests under non-normality}

The tests described previously assume that returns are normally distributed and the error term in Equation (1) is homoskedastic.

However, HF or FOF returns are generally notnormally distributed. In this case, we relax the normality assumption and we use a GMM Wald (Wa) test to adjust for return non-normality:

$$
\begin{aligned}
W_{a}=T & \times \operatorname{vec}\left(\hat{\Theta}^{\prime}\right) /\left[\left(A_{T} \otimes I_{N}\right)\right. \\
& \left.S_{T}\left(A_{T}^{\prime} \otimes I_{N}\right)\right]^{-1} \operatorname{vec}\left(\hat{\Theta}^{\prime}\right) \sim \chi_{2 N}^{2}
\end{aligned}
$$

where the moment condition is

$$
\begin{gathered}
E\left[g_{t}\right]=E(X \otimes E)=0_{N(1+K)}^{\prime} \\
S T=E\left[g_{t}^{\prime} g_{t}\right] \\
A T=\left[\begin{array}{ll}
1+\hat{a}_{1} & -\hat{\mu}_{1} \hat{V}_{11}^{-1} \\
\hat{b}_{1} & -1_{K}^{\prime} \hat{V}_{11}^{-1}
\end{array}\right]
\end{gathered}
$$

\section{Mean-variance intersection tests}

A mean-variance intersection test is equivalent to a test for mean-variance spanning for a specific value of the zero-beta rate. Huberman and Kandel show that the null hypothesis of mean-variance intersection is:

$$
H_{0}: \alpha=R^{0}\left(1_{N}-\beta 1_{K}\right)
$$

where $R_{0}$ is the zero beta of return associated with the mean-variance efficient portfolio at the intersection point. DeRoon and Nijman show that the Wald test for mean-variance intersection is:

$$
W^{\text {intersection }}=T\left(\frac{1+\hat{\theta}_{R}\left(R^{0}\right)^{2}}{1+\hat{\theta}_{R_{1}}\left(R^{0}\right)^{2}}-1\right)
$$

where $\hat{\theta}_{R_{1}}\left(R^{0}\right)$ is the Sharpe ratio of the benchmark assets, and $\hat{\theta}_{R}\left(R^{0}\right)$ is the Sharpe ratio of benchmark plus test assets. DeRoon and Nijman show that the Wald test intersection statistic can easily be interpreted as the percentage increase in Sharpe ratios scaled by the sample size. Hence, the intersection hypothesis is equivalent to the hypothesis that the Jensen performance measure is zero. If there is intersection, then there is no possible improvement in the Sharpe measure to be achieved by including the additional assets $N$ in the investor's portfolio $K$.

For the spanning test, the Wald test statistic can be re-written as

$$
\begin{aligned}
W= & T\left(\frac{1+\hat{\theta}_{R}\left(R^{0}\right)^{2}}{1+\hat{\theta}_{R_{1}}\left(R^{0}\right)^{2}}-1\right) \\
& +T\left(\frac{\left(\hat{\sigma}_{R_{1}}\right)^{2}}{\left(\hat{\sigma}_{R}\right)^{2}}-1\right)
\end{aligned}
$$

where $\hat{\theta}_{R_{1}}\left(R^{0}\right)$ is the Sharpe ratio of the benchmark assets and $\hat{\theta}_{R}\left(\mathrm{R}^{0}\right)$ is the Sharpe ratio 
of benchmark plus test assets. $\hat{\sigma}_{R_{1}}^{2}$ and $\hat{\sigma}_{R}^{2}$ are the global minimum variance of the benchmark assets and that of the benchmark assets plus a HF portfolio, respectively. The equation shows that the spanning test statistic consists of two parts: the first is similar to the intersection statistic test and determined by a change in Sharpe ratios, and the second is determined by the change in the global minimum variance of the portfolios.

To sum up, there is intersection even if there is only one value for which mean-variance investors cannot improve their mean-variance efficient portfolio by including $R_{2, t+1}$ in their investment set. In other words, the mean-variance frontier of $R_{1, t+1}$ intersects $R_{1, t+1}+R_{2, t+1}$. Furthermore, there is spanning if there are no mean-variance investors that can improve their mean-variance efficient portfolio by including $R_{2, t+1}$ in their investment set. In other words, the mean-variance frontier of $R_{1, t+1}$ spans $R_{1, t+1} R_{2, t+1}$.

\section{DATA}

To represent the HF and FOF universes, we choose to use The Credit Suisse First Boston/ Tremont (CSFB/Tremont) Hedge Fund Index and TASS Fund of Hedge Funds Index (TASS FOF), respectively. We also use two benchmark sets. First, we take five size and five book-tomarket portfolios to form 25 value-weighted size and book-to-market portfolios of NYSE, AMEX and NASDAQ stocks. The 25 benchmark portfolios are obtained from French's website. ${ }^{5}$ Panel A of Table 1 presents the summary statistics of the 25 size/book-to-market portfolios. These portfolios show a mean return range from 0.55 to 1.88 per cent and a range of standard deviation from 4.37 to 9.79 per cent.

Second, we enlarge our benchmark set by including international asset classes, bonds and commodities. Thus, our second benchmark includes: Standard \& Poor's 500 for US large capitalisation stocks, Standard \& Poor's SmallCap 600 for US small capitalisation stocks, Morgan Stanley Capital International (MSCI) EAFE for Europe, Australia and Far East stocks, MSCI EM for emerging markets stocks, Goldman Sachs Commodity Index (GSCI) for commodities, Lehman Brothers Aggregate Index for fixed income, and Payden \& Rygel (P\&R) 90 day T-Bill for short term investments. Panel B of Table 1 presents the summary statistics of the second benchmark assets.

\section{EMPIRICAL RESULTS}

\section{Full sample results}

Table 2 reports the MVST results for the period 1994-2004. Based on LM, LR, W and GMM Wa tests, we reject the null hypothesis that the 25 size/book-to-market portfolios as benchmark portfolios (B1) can span the HF portfolio (as represented by the CSFB/Tremont Hedge Fund Index) at the 1 per cent confidence level. Therefore, we suggest that investing in HF does bring diversification benefits for mean-variance investors. However, when we enlarge our investment opportunity set by including fixed income, international assets and commodities (B2), we cannot reject the spanning hypothesis at the 5 per cent level. This result is somewhat to be expected due to the presence of international asset classes, commodities and bonds. Thus, the value added by HF is uncertain and depends upon the opportunity set that is considered.

On the other hand, based on LM, LR and Wa tests, we reject the null hypothesis that the 25 size/book-to-market portfolios as benchmark portfolios can span the FOF portfolio at the 1 
Table 1: Summary statistics of benchmark portfolios (1994-2004)

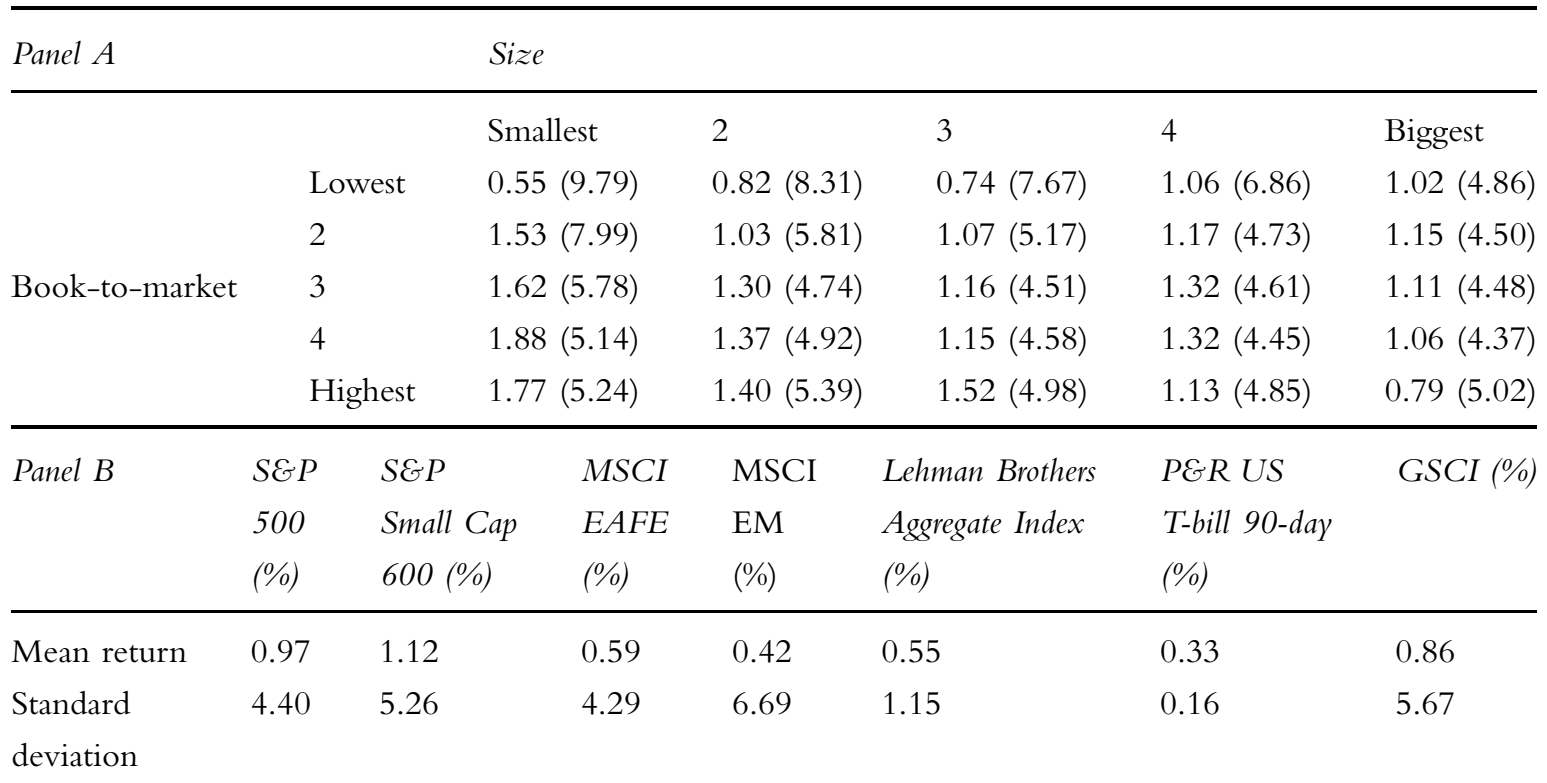

Panel A presents the mean monthly percentage returns and monthly percentage standard deviations (in parentheses) of the 25 size/book-to-market portfolios.

Panel B presents the mean monthly returns and monthly standard deviations of the second benchmark (B2) assets. B2 includes: Standard \& Poor's 500 for US large capitalisation stocks, Standard \& Poor's SmallCap 600 for US small capitalisation stocks, Morgan Stanley Capital International (MSCI) EAFE for Europe, Australia and Far East stocks, MSCI EM for emergent markets stocks, Lehman Brothers Aggregate Index for fixed income, Payden \& Rygel (P\&R) 90-day T-bill for short-term investments and Goldman Sachs Commodity Index (GSCI) for commodities.

per cent confidence level. We also confirm these results using the GMM Wa test allowing for heteroskedasticity. Therefore, we suggest that investing in FOF does bring diversification benefits for mean-variance investors, even when we enlarge our investment opportunity set by including fixed income, international assets and commodities.

To further investigate the sources of the spanning hypothesis rejection, we implement a step-down procedure as suggested by Kan and Zhou. These authors divide the spanning test into two: the first test is related to the tangency portfolio and refers to the restriction on the intercepts of the regression $\left(\alpha=0_{N}\right)$, and the second is related to the global minimumvariance portfolio and refers to the constraint on the estimated coefficients $\left(\delta=1_{N}-\beta 1_{K}=0_{N}\right)$. As sustained by Petrella, ${ }^{6}$ this division is important for investors with a low risk aversion, as they will benefit more from the improvement of the tangency portfolio's characteristics.

Table 3 reports results of the step-down procedure. Panel A of Table 3 reports results for the CSFB/Tremont Hedge Fund Index. We find that, when we use B1, the null hypothesis related to the tangency portfolio is not rejected, while we reject the null hypothesis of the global 
Table 2: Mean-variance spanning tests of hedge funds and funds of hedge funds portfolios (1994-2004)

\begin{tabular}{|c|c|c|c|c|c|c|c|c|}
\hline & \multicolumn{4}{|c|}{ Benchmark (B1) } & \multicolumn{4}{|c|}{ Benchmark (B2) } \\
\hline & \multicolumn{3}{|c|}{$\begin{array}{l}\text { Regression-based } \\
\text { mean-variance } \\
\text { spanning tests }\end{array}$} & \multirow{2}{*}{$\begin{array}{l}\text { GMM } \\
W a \\
\text { test } \\
W a\end{array}$} & \multicolumn{3}{|c|}{$\begin{array}{l}\text { Regression-based } \\
\text { mean-variance } \\
\text { spanning tests }\end{array}$} & \multirow{2}{*}{$\begin{array}{l}\text { GMM } \\
W a \\
\text { test } \\
W a\end{array}$} \\
\hline & $L M$ & $L R$ & $W$ & & $L M$ & $L R$ & $W$ & \\
\hline \multicolumn{9}{|l|}{ Panel $A$} \\
\hline CSFB/Tremont & 7.663 & 11.470 & 18.273 & 8.014 & 5.076 & 5.176 & 5.279 & 4.454 \\
\hline Hedge Fund Index & $(0.005)$ & $(0.000)$ & $(0.000)$ & $(0.004)$ & $(0.079)$ & $(0.075)$ & $(0.071)$ & $(0.108)$ \\
\hline \multicolumn{9}{|l|}{ Panel B } \\
\hline TASS FOF Index & $\begin{array}{l}107.46 \\
(<0.000)\end{array}$ & $\begin{array}{l}222.10 \\
(<0.000)\end{array}$ & $\begin{array}{l}578.12 \\
(<0.000)\end{array}$ & $\begin{array}{l}462.79 \\
(<0.000)\end{array}$ & $\begin{array}{l}7.073 \\
(0.029)\end{array}$ & $\begin{array}{l}7.269 \\
(0.026)\end{array}$ & $\begin{array}{l}7.473 \\
(0.023)\end{array}$ & $\begin{array}{l}4.821 \\
(0.089)\end{array}$ \\
\hline
\end{tabular}

The test portfolios are the CSFB/Tremont Hedge Fund and TASS Funds of Hedge Funds Indexes. Benchmark (B1) includes 25 size/book-to-market portfolios that are constructed by all of the firms trading on the NYSE, AMEX or NASDAQ. Benchmark (B2) includes: Standard \& Poor's 500 for US large capitalisation stocks, Standard \& Poor's SmallCap 600 for US small capitalisation stocks, Morgan Stanley Capital International (MSCI) EAFE for Europe, Australia and Far East stocks, MSCI EM for emergent markets stocks, Goldman Sachs Commodity Index (GSCI) for commodities, Lehman Brothers Aggregate Index for fixed income, and Payden \& Rygel (P\&R) 90-day T-bill for short-term investments. LM, LR and W represent the asymptotic Lagrange multiplier, likelihood ratio and Wald tests, respectively. Wa is the GMM Wald test allowing for heteroskedasticity. The $p$-values are in parentheses.

minimum-variance portfolio at the 1 per cent confidence level. Thus, investing in a HF portfolio (as represented by the CSFB/Tremont Hedge Fund Index) improves the global minimum-variance portfolio but not the tangency portfolio. However, when we use B2, we reject neither of the tests. Investors who already hold a diversified portfolio with international assets, bonds and commodities do not improve their investment opportunity set by adding HF portfolios.

Panel B of Table 3 reports results for the TASS FOF. We find that, when we use B1, the null hypothesis related to the tangency portfolio is not rejected, while we reject the null hypothesis of the global minimum-variance portfolio at the 1 per cent confidence level. Thus, investing in a FOF portfolio improves the global minimumvariance portfolio but not the tangency portfolio. We also confirm this result using B2.

\section{Mean-variance spanning tests in terms of performance measures}

The purpose of this section is to examine the added value of HF and FOF in terms of the 
Table 3: Mean-variance intersection and spanning tests of Hedge funds and funds of Hedge funds portfolios (1994-2004)

Step-down tests

\begin{tabular}{llll}
\hline Benchmark (B1) & & $\frac{\operatorname{Benchmark}(B 2)}{W_{1}}$ & $W_{2}$ \\
\hline$W_{1}$ & $W_{2}$ &
\end{tabular}

Panel $A$

CSFB/Tremont Hedge Fund Index 0.683 (0.710) $\quad 9.604(0.008) \quad 0.0001(1.000) \quad 3.513(0.173)$

Panel B

TASS FOF Index $\quad 1.138(0.566) \quad 462.795(<0.000) \quad 0.004(0.997) \quad 4.821(0.089)$

The test portfolios are the CSFB/Tremont Hedge Fund and TASS Funds of Hedge Funds Indexes. Benchmark (B1) includes 25 size/book-to-market portfolios that are constructed by all of the firms trading on the NYSE, AMEX or NASDAQ. Benchmark (B2) includes: Standard \& Poor's 500 for US large capitalisation stocks, Standard \& Poor's SmallCap 600 for US small capitalisation stocks, Morgan Stanley Capital International (MSCI) EAFE for Europe, Australia and Far East stocks, Morgan Stanley Capital International (MSCI) EM for emergent markets stocks, Goldman Sachs Commodity Index GSCI for commodities, Lehman Brothers Aggregate Index for fixed income and Payden \& Rygel (P\&R) 90-day T-bill for short-term investments. The first test $\left(\mathrm{W}_{1}\right)$ is a Wald test of $\mathrm{H}_{0}: \alpha=0_{N}$, and the second test $\left(\mathrm{W}_{2}\right)$ is a Wald test of $\mathrm{H}_{0}: \delta=1_{N}-\beta 1 K=0_{N}$ conditional on $\alpha=0_{N}$. The $p$-values are in parentheses.

Sharpe ratio and Jensen's alpha, using MVSTs. Bekaert and Urias ${ }^{7}$ point out that the change in the Sharpe ratio of the tangency portfolio measures the economic importance of the shift in the efficient frontier. As the Sharpe ratio of the tangency portfolio gives the largest mean return per unit of standard deviation, a difference between the Sharpe ratios computed for the benchmark assets $(K)$ and the $K+N$ (new assets) indicates that investors can enhance their returns per unit of risk by investing in the additional $N$ assets.

Further, in addition to using the change in the maximum Sharpe ratio, we use Jensen's alpha as a measure of portfolio efficiency. To do so, we conduct mean-variance intersection tests that are, as previously shown, equivalent to Jensen's alpha measure of portfolio performance.

Panel $A$ and $B$ of Table 4 report the results for the mean-spanning tests (using the Sharpe ratio) and the mean-variance intersection tests (equivalent to Jensen's alpha) for the CSFB/ Tremont Hedge Fund Index and the TASS FOF indexes, respectively. First, we find that when we consider B1 as the benchmark portfolio, we fail to reject the mean-variance intersection hypothesis for CSFB/Tremont Hedge Fund Index. Thus, there is no evidence of abnormal performance for HF portfolio relative to the benchmark B1. However, this conclusion is less evident when we consider B2 as a benchmark portfolio. 
Table 4: Mean-variance intersection tests and mean-variance spanning tests (in terms of sharpe ratios) of hedge funds and funds of hedge funds portfolios (1994-2004)

\begin{tabular}{|c|c|c|c|c|}
\hline & \multicolumn{2}{|l|}{ Benchmark (B1) } & \multicolumn{2}{|l|}{ Benchmark (B2) } \\
\hline & Intersection & Spanning & Intersection & Spanning \\
\hline & hypothesis & hypothesis & hypothesis & hypothesis \\
\hline & $W^{\text {Intersection }}$ & $W^{\text {Spanning }}$ & $W^{\text {Intersection }}$ & $W^{\text {Spanning }}$ \\
\hline \multicolumn{5}{|l|}{ Panel $A$} \\
\hline CSFB/Tremont Hedge & $2.564(0.109)$ & $16.090(0.000)$ & $29.361(0.001)$ & $23.590(0.009)$ \\
\hline \multicolumn{5}{|l|}{ Fund Index } \\
\hline \multicolumn{5}{|l|}{ Panel B } \\
\hline TASS FOF Index & $65.978(<0.000)$ & $257.908(<0.000)$ & $0.070(0.791)$ & $3.729(0.053)$ \\
\hline
\end{tabular}

The test portfolios are the CSFB/Tremont Hedge Fund and TASS Funds of Hedge Funds Indexes. Benchmark (B1) includes 25 size/book-to-market portfolios that are constructed by all of the firms trading on the NYSE, AMEX or NASDAQ. Benchmark (B2) includes: Standard \& Poor's 500 for US large capitalisation stocks, Standard \& Poor's SmallCap 600 for US small capitalisation stocks, Morgan Stanley Capital International (MSCI) EAFE for Europe, Australia and Far East stocks, Morgan Stanley Capital International (MSCI) EM for emergent markets stocks, Goldman Sachs Commodity Index GSCI for commodities, Lehman Brothers Aggregate Index for fixed income and Payden \& Rygel (P\&R) 90-day T-bill for short-term investments. W represents the asymptotic Wald test. The $p$-values are in parentheses.

Panel B of Table 4 reports the results for the mean-spanning tests and the mean-variance intersection tests for the FOF portfolio. We find that, when we consider B1 as the benchmark portfolio, we reject the mean-variance intersection hypothesis. In other words, there is a significant abnormal performance on FOF portfolio relative to a set of 25 size/book-tomarket portfolios as benchmarks. However, we fail to reject the mean-variance intersection hypothesis for FOF portfolio when we consider B2 as a benchmark portfolio.

Overall, we conclude that including HF or FOF portfolio in a set of benchmark portfolios (US stocks only) does provide an extra return for a unit increase in standard deviation. However, this conclusion is less evident when we consider a diversified benchmark.

\section{CONCLUSION}

In this paper, we examine whether investors can improve their investment opportunity set by adding a HF or fund of HF portfolio to different sets of benchmark portfolios. We apply a battery of mean-variance spanning tests using data from 1994 to 2004. To the best of our knowledge, our study is the first to use these tests in the HF or FOF context.

When we consider benchmark portfolios sorted by firm size and book-to-market ratio, we find that HFs, as an asset class, improve the 
mean-variance frontier set. However, the improvement comes mainly from a leftward shift of the global minimum-variance portfolio rather than the tangency portfolio. These results are especially important to low risk investors who are looking for alternative assets to enlarge the minimum-variance frontier. Additionally, we find that investors who already hold a diversified portfolio with international assets, bonds and commodities do not improve their investment opportunity set by adding HF portfolios. On the other hand, results show that investing in FOF does bring diversification benefits for meanvariance investors, even when we enlarge our investment opportunity set by including fixed income, international assets and commodities.

Further, to investigate the economic importance of the shift in the efficient frontier that results from adding HF or FOF, we conduct further tests: mean-variance spanning tests using Sharpe ratios and mean-variance intersection tests that are equivalent to the Jensen's alpha measure of portfolio performance. Our general conclusion is that including HF or FOF portfolios to a set of benchmark portfolios (US stocks only) provides an extra return for a unit increase in standard deviation. However, this conclusion is less evident when we consider an internationally diversified portfolio as a benchmark.

These results confirm Amin and $\mathrm{Kat}^{8}$ observations. These authors investigate the diversification effects that occur when combining HF with stocks and bonds. They show that HF do not mix too well with equity and that including HF in a traditional investment portfolio not only improve portfolio's meanvariance characteristics, but also lead to significantly lower skewness as well as higher kurtosis. This is an important result giving that most institutional investors continue to allocate growing percentage of their assets to HF. Furthermore, we confirm that not only FOF allow for institutional investors to have access to the best HF managers, but they do provide added diversification. The HF and the funds of HF universe is evolving rapidly and much remains to be done to better understand the importance of these alternative investments to asset allocation.

\section{References}

1 Brown, S. J., Goetzmann, W. N. and Liang, B. (2004) 'Fees on Fees in Funds of Funds', Working Paper, Yale ICF No. 02-33.

2 DeRoon, F. A. and Nijman, T. E. (2001) 'Testing for Mean-Variance Spanning: A Survey', Journal of Empirical Finance, Vol. 8, No. 2, pp. 111-155.

3 Kan, R. and Zhou, G. (2001). 'Test of Mean-Variance Spanning, Working Paper, University of Toronto.

4 Huberman, G. and Kandel, S. (1987) 'Mean-Variance Spanning', Journal of Finance, Vol. 42, No. 4, pp. 873-888.

5 French, K. R. (2005). 'Data Library, http:// mba.tuck.dartmouth.edu/pages/faculty/ken.french/ data_library.html.

6 Petrella, G. (2005) 'Are Euro Area Small Cap Stocks an Asset Class? Evidence from Mean-Variance Spanning Tests', European Financial Management, Vol. 11, No. 2, pp. 229-253.

7 Bekaert, G. and Urias, M. (1996) 'Diversification Integration and Emerging Market Closed-End Funds', Journal of Finance, Vol. 51, No. 3, pp. 835-869.

8 Amin, G. and Kat, H. (2002) 'Stocks, Bonds and Hedge Funds: Not a Free Lunch!', Working Paper, ISMA Centre, University of Reading.

\section{Appendix A}

\section{Asymptotic and finite test statistics for mean-variance spanning tests}

Following Huberman and Kandel, ${ }^{4}$ the null hypothesis of 'spanning' is:

$$
H_{0}: \alpha=0_{N}, \delta=1_{N}-\beta 1_{K}=0_{N} .
$$

We can write the null hypothesis as $\Theta=[\alpha$ $\delta]^{\prime}=\mathrm{O}_{2 \times N}=A B-C$, 
where

$$
A=\left[\begin{array}{cc}
1 & 0^{\prime}{ }_{K} \\
0 & -1_{K}^{\prime}
\end{array}\right] \text { and } C=\left[\begin{array}{c}
0^{\prime}{ }_{K} \\
-1_{K}^{\prime}
\end{array}\right]
$$

The distribution of the null hypothesis is $\operatorname{vec}(\hat{\Theta}) \sim N\left(\operatorname{vec}\left(\Theta^{\prime}\right), A\left(X^{\prime} X\right)^{-1} A^{\prime} \otimes \Sigma\right)$. By defining

$$
\begin{aligned}
\hat{G} & =T A\left(X^{\prime} X\right)-1 A^{\prime} \\
& =\left[\begin{array}{cc}
1+\hat{\mu}_{1^{\prime}}^{\prime} \hat{V}_{11}^{-1} \hat{\mu}_{1^{\prime}} & \hat{\mu}^{\prime}{ }^{\prime} \hat{\mathrm{V}}_{11}^{-1} 1_{K} \\
\hat{\mu}^{\prime}{ }_{1^{\prime}} \hat{\mathrm{V}}_{11}^{-1} 1_{K} & 1^{\prime}{ }_{K} \hat{\mathrm{V}}_{11}^{-1} 1_{K}
\end{array}\right]
\end{aligned}
$$

and

$$
\hat{H}=\hat{\Theta} \hat{\sum}^{-1} \hat{\Theta}=\left[\begin{array}{ll}
\hat{\alpha}^{\prime} \hat{\sum}^{-1} \hat{\alpha} & \hat{\alpha}^{\prime} \hat{\sum}^{-1} \hat{\delta} \\
\hat{\alpha}^{\prime} \hat{\sum}^{-1} \hat{\delta} & \hat{\delta}^{\prime} \hat{\sum}^{-1} \hat{\delta}
\end{array}\right]
$$

we then denote the two eigenvalues of the matrix, $\hat{H} \hat{G}^{-1}$, by $\lambda_{1}$ and by $\lambda_{2}$. 